\title{
Mismos caminos, distintos destinos. Explorando el efecto de las trayectorias educativas y del origen social en las transiciones hacia la educación postobligatoria'
}

\author{
Same Paths, Different Destinations. Exploring the Effect of Educational \\ Trajectories and Social Background on Transitions to Post-compulsory \\ Education
}

Alba Castejón, Alejandro Montes y Martí Manzano²

\begin{abstract}
Resumen
Las transiciones a la educación postobligatoria son un elemento clave para comprender las desigualdades educativas y sociales. La elección de los estudios postobligatorios por parte de los y las jóvenes está mediada por patrones de desigualdad social, así como por sus trayectorias y experiencias escolares previas. Partiendo de este marco, este artículo responde al objetivo de comprender de qué forma las trayectorias escolares de los y las jóvenes en la etapa de la educación obligatoria contribuyen a explicar sus elecciones acerca del itinerario postobligatorio. Más allá de un análisis que incorpore los factores descriptivos de las trayectorias escolares -como la repetición de curso o el rendimiento académico-el análisis incorpora también las experiencias de los y las jóvenes en su paso por la escuela. Para ello se utilizan los datos de un cuestionario realizado durante el curso 2018-2019 a N=1318 jóvenes en su primer curso de educación postobligatoria. Se ha realizado un análisis factorial y un posterior análisis de conglomerados -clúster-, que resultan en la construcción de cuatro tipos de trayectorias escolares: los/as «buenos/as», los/ as «esforzados/as», los/as «liantes/as», y los/as «desplazados/as». Los resultados muestran cómo estas trayectorias escolares, mediadas por el origen social, influyen en las elecciones postobligatorias. En definitiva, el artículo pone de relieve la importancia ejercida por la trayectoria escolar y aporta una nueva evidencia a la desmitificación de la retórica existente sobre la «libre elección» en la transición a los estudios postobligatorios.
\end{abstract}

\section{Palabras clave}

Transición educativa, trayectoria escolar, experiencia escolar, educación postobligatoria, elección postobligatoria.

\section{Abstract}

Transitions to post-compulsory education are a key element in understanding educational and social inequalities. The choice of post-compulsory education is mediated by patterns of social inequality, as well as by school trajectories and previous educational experiences. Based on this framework, this article aims at understanding how school trajectories of young people in the compulsory education help to explain their choices about post-compulsory itinerary. Beyond an analysis that incorporates the descriptive factors of school trajectories - such as grade retention or youngsters' academic performance - the analysis also incorporates the experiences of young people at school. In doing so, we used the data from a questionnaire carried out during the 2018-2019 academic year to $\mathrm{N}=1,318$ young people in their first post-compulsory education course. We carried out a factorial analysis and a subsequent cluster analysis, which resulted in the construction of four types of school trajectories: the «good students», the «hard-working», the «misbehaved» and the «displaced». Results show how these school trajectories, mediated by social origin, influence post-compulsory choices. The article highlights the importance of the school career and provides new evidence for the demystification of the existing rhetoric about "free choice" in the transition to post-compulsory studies.

\section{Keywords}

Educational transition, school pathways, school experience, upper-secondary education, upper-secondary choice.

\section{Cómo citar/Citation}

Castejón, Alba; Montes, Alejandro; Manzano, Martí (2020). Mismos caminos, distintos destinos. Explorando el efecto de las trayectorias educativas y del origen social en las transiciones hacia la educación postobligatoria. Revista de Sociología de la Educación-RASE, 13 (4), 507-525. http://dx.doi.org/10.7203/RASE.13.4.18030

\footnotetext{
Este estudio ha sido financiado en el marco del proyecto «Edupost16. La construcción de las oportunidades educativas post-16. Un análisis de las transiciones a la educación secundaria post-obligatoria en contextos urbanos» (http://edupost16.es), financiado por el Ministerio de Economía y Competitividad Español para el período 2016-19 (CSO2016-80004-P).

2 Alba Castejón, Universitat Autònoma de Barcelona, alba.castejon@uab.cat; Alejandro Montes, Universitat Autònoma de Barcelona, alejandro.montes@uab.cat; Martí Manzano, Universitat Autònoma de Barcelona, marti.manzano@uab.cat.
} 


\section{Introducción}

Las trayectorias y transiciones educativas han copado, a lo largo de las últimas décadas, una parte importante del campo de estudio de la sociología de la educación (Pallas, 2003; Walther, et al., 2015; Cuconato, et al., 2015). Su relevancia radica en el papel que la educación, como institución social, tiene tanto en los procesos de estratificación y reproducción social como en los procesos de socialización de los y las jóvenes. Por un lado, las trayectorias educativas se han mostrado centrales para explicar los destinos sociales de los individuos, mientras que, a su vez, estas trayectorias están marcadas por el origen social de los mismos (Pallas, 2003). En este sentido, las transiciones a la educación postobligatoria son un elemento clave para entender las desigualdades educativas y sociales, y, asimismo, están condicionadas por una estructura de oportunidades desigual. Por consiguiente, en el presente artículo la transición a la educación postobligatoria se concibe teniendo en cuenta que la agencia de los y las jóvenes se encuentra delimitada tanto por un conjunto de condicionantes estructurales, como la clase social, el género, o el origen migrante, como institucionales, como la escuela (Bernardi y Requena, 2010; Cuconato y Walther, 2015; Elias y Daza, 2017; Furlong, 2009; Jacovkis, Montes y Manzano, 2020; Pallas, 2003). La institución escolar es, pues, un elemento central en la construcción de trayectorias educativas y en las elecciones de los y las jóvenes (Tarabini e Ingram, 2018).

Partiendo de este marco, el artículo tiene como objetivo identificar la transición a la educación postobligatoria analizando específicamente su relación con las trayectorias educativas de los y las jóvenes en su paso por la educación obligatoria, y de qué forma esta relación está mediada por variables de carácter estructural como la clase, el género o el origen migrante. En el sistema educativo español, la transición a la educación postobligatoria representa la primera en la que los y las jóvenes optan, de forma efectiva, entre diferentes itinerarios formativos: por un lado, el bachillerato, el itinerario académico; y, por otro lado, la formación profesional de grado medio (CFGM), el itinerario profesional. Así pues, los y las estudiantes de 16 años, en el término de la educación obligatoria, pueden escoger entre estos dos itinerarios, o bien salir del sistema formativo y dirigirse al mundo laboral.

Concretamente, el artículo analiza las transiciones a la educación postobligatoria según las trayectorias educativas previas, que se definen tanto en términos objetivos-entendidos como aquellos eventos o situaciones específicas que suceden en el marco de las trayectorias, como pueden ser la repetición de curso, el rendimiento académico, o episodios de abandono escolar-como en términos subjetivos-entendidos como las experiencias y vivencias de los jóvenes acerca de dichos eventos o situaciones, esto es, las formas en como los y las jóvenes viven y se relacionan con la institución escolar-. Asimismo, se pretende identificar de qué forma los condicionantes estructurales -la clase social, el género y el origen migrante- median entre las trayectorias educativas previas y las transiciones a la educación postobligatoria.

Para ello, se han analizado los datos de un cuestionario distribuido a 1318 jóvenes de Barcelona que están cursando el primer año de educación secundaria postobligatoria. A partir de las respuestas de 915 de estos y estas jóvenes, el artículo se plantea los siguientes objetivos: primero, establecer una tipología de trayectorias escolares previas, teniendo en cuenta variables tanto objetivas (rendimiento académico, repetición de curso) como subjetivas (como el sentimiento de utilidad de la escuela o el bienestar); segundo, establecer las correspondencias entre las trayectorias identificadas y los itinerarios de educación postobligatoria; y tercero, identificar si existen diferencias entre las trayectorias previas y los itinerarios de educación postobligatoria en función de variables como la clase social, el género o el origen migrante, en 
tanto que éstas ejercen un papel mediador entre las trayectorias escolares previas y la distribución de los y las jóvenes en los diferentes itinerarios.

El artículo se organiza de la siguiente manera. Después de este apartado introductorio, se presenta una breve revisión teórica y empírica sobre el concepto de trayectoria educativa, recogiendo especialmente aquellas aportaciones que sitúan la institución escolar y la experiencia de los y las jóvenes en el centro del análisis y la vinculan con la transición a la educación postobligatoria. En segundo lugar, se presenta el planteamiento metodológico del artículo, señalando la muestra y los instrumentos, así como la estrategia de análisis de los datos. En tercer lugar, se presentan los resultados del estudio, que comprenden una tipología de trayectorias educativas previas, su relación con el destino de educación postobligatoria, y su relación con las variables de perfil social de la muestra. En último lugar, el artículo recoge las principales conclusiones que ponen de relieve el papel de la trayectoria escolar en la construcción de las transiciones a la educación postobligatoria y la importancia de los condicionantes estructurales en dicha construcción.

\section{Las trayectorias educativas como objeto de estudio}

El estudio de las trayectorias - vitales, educativas- ha sido un objeto central en la investigación sociológica. Estas, en términos generales, se pueden definir como la secuencia de acontecimientos y estadios que un individuo recorre a lo largo de su ciclo vital (Pallas, 2003). En el caso de la educación, las trayectorias se refieren a los eventos educativos y a las transiciones entre etapas y hacia el mundo laboral (Cuconato y Walther, 2015; Pallas, 2003). El interés en su investigación recae tanto en su capacidad explicativa como determinante de los acontecimientos que se suceden a lo largo del ciclo vital -en términos de estratificación social, por ejemplo-, como fenómeno a ser explicado en sí mismo -en relación con los determinantes que explicarían diferentes trayectorias educativas, por ejemplo-. Más allá de las investigaciones empíricas, diversos autores han hecho aportaciones acerca del propio concepto de trayectoria, diferenciándolo de conceptos afines como «transición» o «itinerario», y señalando los cambios que, a lo largo de las últimas décadas, ha habido en las trayectorias de los y las jóvenes (Furlong 2009; Pallas, 2003). A continuación, para situar el enfoque teórico del presente artículo, presentamos brevemente algunos apuntes conceptuales sobre las trayectorias educativas.

\subsection{Una perspectiva relacional de las trayectorias educativas}

Las trayectorias y transiciones educativas se han explicado de diferentes formas a lo largo de las últimas décadas, mediante el uso de diferentes «metáforas» (Furlong, 2009). Los cambios en las formas en que se conciben las trayectorias de los y las jóvenes responden, tal y como explica el autor, a dos elementos principales: por un lado, a cambios en las experiencias de los jóvenes, condicionados, por ejemplo, por variaciones en el mercado laboral o por los cambios normativos de los sistemas educativos (Wyn y Dwyer, 2002); y, por otro lado, a las tendencias teóricas presentes en cada momento histórico y a su evolución. Esto ha llevado al desarrollo de diferentes corrientes que, a la hora de explicar las trayectorias, difieren en el peso que otorgan tanto a la estructura como a la agencia. El enfoque de este artículo se basa, siguiendo la propuesta de autores como Cuconato y Walther (2015), Furlong (2009) o Pallas (2003), en una concepción relacional y contextualizada de las trayectorias educativas que asume que estas se explican por interacciones complejas entre diferentes actores y factores, y que tienen lugar en contextos específicos. En este sentido, se parte de un enfoque que, a la hora de concebir las trayectorias educativas, tiene en cuenta las relaciones que se producen entre condicionantes 
estructurales, mediaciones institucionales y estrategias subjetivas. Esto es, una perspectiva que asume que existe una estructura de oportunidades que refleja las posiciones y desigualdades estructurales en las biografías, en las experiencias y en las elecciones de los y las jóvenes, y en las posibilidades u obstáculos que encuentran a lo largo de sus trayectorias educativas y vitales. Así, desde este enfoque, en el análisis de las trayectorias es clave tener en cuenta, como señala Pallas (2003:168) que «el origen social influencia las transiciones educativas y ocupacionales, tanto al estructurar las elecciones que hacen los individuos, como al dar forma a las estructuras en las que los individuos pueden ejercer las elecciones».

A nivel empírico, la elección que los y las jóvenes ejercen al finalizar la educación obligatoria -en el caso español, a los 16 años, al finalizar la secundaria inferior- está claramente marcada, tal y como han reseñado numerosos estudios, por patrones de estratificación social (Bernardi y Requena, 2010; Bernardi y Cebolla-Boado, 2014; Calero, 2008; Elias y Daza, 2017; Jacovkis, Montes y Manzano, 2020). En este sentido, estas investigaciones señalan que la distribución de los y las jóvenes en los diferentes itinerarios -académico o profesional-, así como las tasas de participación en estudios postobligatorios, entre otros outcomes, es desigual en función de la clase social de origen, el género o el origen migrante, e identifican diversas dimensiones que explicarían dichas desigualdades, entre los cuales se encuentran el rendimiento académico, las aspiraciones y expectativas educativas, o los significados y prestigios de cada uno de los itinerarios formativos.

\subsection{El papel del sistema educativo y de la institución escolar en la configuración de trayectorias educativas}

La investigación previa ha demostrado que la institución educativa contribuye a la configuración de estos mecanismos explicativos y que, además, tiene un impacto diferencial según el origen social de los y las jóvenes, por lo que juega un papel mediador entre el origen social y sus elecciones (Cuconato, et al., 2015; Tikkanen, et al., 2015; Walther et al., 2015). En este sentido, el papel de la escuela y del sistema educativo, como parte de la estructura de oportunidades, es central en la configuración de las trayectorias formativas. Aquí es especialmente útil la diferencia analítica que señala Pallas (2003) entre trayectorias e «itinerarios»-pathways, en inglés-. Aunque ambos se puedan describir como una secuencia de etapas y de transiciones, el autor apunta que las trayectorias son atributos individuales, mientras que los «itinerarios» son atributos de un sistema social, los caminos trazados en los mapas del sistema. Los «itinerarios», desde este punto de vista, sitúan el foco de análisis en las estructuras, esto es, en las limitaciones, los incentivos y las oportunidades de elección que el sistema ofrece a los y las jóvenes.

En este sentido, ciertas características de los sistemas educativos, como el grado de estratificación interna o de selección de los y las estudiantes, o las posibilidades de acceso a las diferentes vías formativas o de continuidad académica posterior, marcan los posibles «itinerarios» de los y las jóvenes y, por lo tanto, son factores clave en la explicación de las desigualdades en las trayectorias educativas (Gamoran, 1992; Grytnes, 2011; Oakes, 1985; Tarabini y Jacovkis, 2019). Son elementos que, en palabras de Pallas (2003), pueden cerrar algunas puertas y abrir otras.

Asimismo, se ha mostrado en repetidas ocasiones que ciertos dispositivos y prácticas escolares, como el agrupamiento por niveles o la repetición de curso, tienen impactos en las trayectorias formativas posteriores de los y las jóvenes, no solamente en términos materiales -por ejemplo, en el grado de adquisición de competencias-(Parsons y Hallam, 2014; Slavin, 1990; entre otros), sino también en 
términos simbólicos a través de la construcción del autoconcepto y de la configuración de expectativas e imaginarios futuros (Castejón, 2017; Oakes, 1985; Pàmies, 2013). Los dispositivos y prácticas de orientación escolar, así como las expectativas del profesorado, son también factores que inciden en la construcción desigual de trayectorias (Bonizzoni et al., 2014; Grytnes, 2011; Van Houtte y Demanet, 2016; Tarabini, Castejón y Curran, 2020). En definitiva, la institución educativa, tanto a nivel sistémico como a nivel de centro, resulta central para comprender las experiencias, las decisiones y las trayectorias educativas de los y las jóvenes.

\subsection{La experiencia escolar como elemento central de las trayectorias educativas}

La experiencia escolar - esto es, las formas en como los y las jóvenes viven y se relacionan con la institución escolar, dan significado a la educación y tejen relaciones en el seno de esta- se ha señalado en diversas ocasiones como una variable clave en la definición de las trayectorias educativas. Diversos estudios han centrado su interés en analizar de qué manera la experiencia escolar tiene un impacto o puede contribuir a explicar diferentes trayectorias educativas, aunque el foco de estudio de muchos de ellos ha sido estudiar la relación entre dicha experiencia y el abandono escolar, más que en la transición en sí misma (Curran, 2017; García y Sánchez, 2020; Tarabini, 2019; entre otros). Estas investigaciones parten del supuesto de que la complejidad y la diversidad de procesos que conducen al abandono escolar se pueden explicar por las formas en cómo los y las jóvenes se vinculan con su proceso educativo, identificando diferentes perfiles o tipos de jóvenes en función de dicha experiencia y de sus actitudes hacia la escuela.

Una tipología clásica sobre las actitudes escolares es la propuesta por Bernstein (1988), en la que el autor distingue cuatro tipos ideales de actitudes escolares (la adhesión, la acomodación, la disociación y la resistencia) según el posicionamiento de los y las jóvenes en relación con el orden instrumental de la escuela (esto es, sus actitudes frente el aprendizaje formal, la utilidad que otorgan a la educación o sus expectativas académicas) y el orden expresivo (las actitudes de los y las jóvenes frente a los valores, las normas y las pautas de conducta transmitidas y esperadas por la institución escolar). Otra tipología, ésta desarrollada en el contexto español, es la propuesta por Bonal et al. (2003) en su investigación sobre las formas de apropiación escolar, en la que identifican diferentes tipos de jóvenes según sus posiciones y disposiciones hacia la escuela: los que tienen éxito, los «justitos», los disociados y los resistentes. En un reciente artículo, García y Sánchez (2020) hacen un repaso de las principales tipologías de jóvenes que la literatura ha identificado en relación con la experiencia escolar y el abandono educativo, y en su análisis muestran la heterogeneidad de perfiles de jóvenes que abandonan sus estudios antes de finalizar la educación secundaria postobligatoria: los aplicados, los instrumentales, los absentistas y los disruptivos.

Como han mostrado muchas de estas investigaciones, las formas de vivir y experimentar la escuela no son independientes del origen social los individuos. Por ello, la articulación de las condiciones objetivas y las dimensiones subjetivas en las que se desarrolla el paso por la institución escolar es un elemento central en el análisis de, entre otros aspectos, las trayectorias educativas (Bonal et al., 2003; Grytnes, 2011; Walther et al., 2015). En otras palabras, la trayectoria educativa se considera una construcción a partir de la experiencia escolar vivida, que incorpora tanto aspectos estructurales como la significación que estos adoptan para el sujeto. En este sentido, más allá de los acontecimientos que ocurren durante la escolarización -como la repetición de curso, las transiciones entre etapas, los cam- 
bios de centro educativo o los propios resultados académicos-, la dimensión subjetiva se ha mostrado clave no solamente para comprender las actitudes y trayectorias escolares de los y las jóvenes, sino también en la comprensión y definición de sus oportunidades educativas (Dubet y Martucelli, 1998).

\section{Planteamiento metodológico}

El análisis que se plantea en este artículo tiene como objetivo identificar de qué manera la transición a la educación postobligatoria está relacionada con las trayectorias educativas de los y las jóvenes en su paso por la educación obligatoria, y de qué forma esta relación está mediada por variables de carácter estructural como la clase, el género o el origen migrante. En comparación con otras tipologías propuestas en la literatura, la clasificación de las trayectorias educativas que se propone en el presente análisis incorpora, además de los elementos subjetivos de dichas trayectorias (esto es, la experiencia de los y las jóvenes en su paso por la institución escolar), elementos objetivos, tales como las notas o las repeticiones de curso.

Este artículo forma parte de un proyecto de investigación más amplio que tiene como objetivo general entender los factores que explican las transiciones a la educación secundaria postobligatoria en contextos urbanos. En el marco de este proyecto se han realizado entrevistas con diferentes actores educativos, cuestionarios a jóvenes y observaciones de jornadas de puertas abiertas. Este artículo se basa en la información recogida mediante un cuestionario a 1318 jóvenes de ocho centros educativos de Barcelona.

\section{1. Muestra e instrumento}

La selección de los centros donde se desarrolló el trabajo de campo se basó principalmente en su oferta educativa, en su ubicación geográfica y en su composición social. Respecto a la oferta, se seleccionaron centros que, además de educación secundaria obligatoria, ofrecieran los dos itinerarios de la etapa secundaria postobligatoria (Bachillerato y FP de grado medio). Más allá de la oferta, también se tuvo en cuenta su composición social y su ubicación geográfica, y se seleccionaron centros que permitiesen garantizar una cierta diversidad: centros compuestos por alumnado de clase acomodada o media, centros con alumnado de clase trabajadora y también centros con altas concentraciones de alumnado en riesgo de exclusión social. De los 8 centros seleccionados, uno es de titularidad privada y los demás son públicos.

Como parte del proyecto general de investigación, se elaboró un cuestionario que tenía como objetivo recoger información sobre la elección de estudios postobligatorios por parte de los y las jóvenes, pero también acerca de sus experiencias durante la educación obligatoria y sobre otros aspectos como su origen social. Concretamente, el cuestionario se organizó en cuatro bloques. La primera parte introducía el cuestionario y recogía datos básicos como la edad, el género o la situación educativa actual. En segundo lugar, un bloque recogía información sobre la trayectoria educativa durante la educación obligatoria y los motivos de elección de los estudios postobligatorios que actualmente cursan. El tercer bloque agrupaba preguntas sobre las concepciones que los y las jóvenes tienen sobre las diferentes vías postobligatorias, así como sus expectativas y aspiraciones de futuro. El último bloque recogía información sobre el origen social de los y las jóvenes, tal como la ocupación y el nivel de estudios de sus progenitores, así como su lugar de nacimiento.

El cuestionario se distribuyó para todo el alumnado de primer curso de educación postobligatoria de los ocho centros entre octubre y diciembre de 2018, para evitar perder parte de la muestra al alum- 
nado que abandona al inicio del curso académico. Pese a ser un cuestionario online, éste se realizó supervisado presencialmente por al menos un investigador o investigadora del proyecto en las aulas de informática de cada uno de los centros. El total de respuestas obtenidas fue de 1318. Debe tenerse en cuenta que, para el presente análisis, se han excluido las personas que finalizaron la educación secundaria obligatoria hace más de 10 años, que la realizaron en un país que no fuese España y los casos que estadísticamente presentaban valores perdidos en las principales variables de análisis. Consecuentemente, el total de casos que forma la muestra de este artículo es de 915 alumnos y alumnas, de los cuales el 550 hacen Bachillerato (el 60,1\%) y 365 hacen un Ciclo Formativo de Grado Medio (CFGM) (el 39,9\%).

\subsection{Variables y estrategia analítica}

Para dar respuesta al objetivo general, el análisis se plantea alrededor de una variable dependiente (itinerario postobligatorio) y diversas variables independientes (trayectoria educativa y variables de perfil social).

La variable dependiente es el «itinerario de educación postobligatoria» y se refiere a los estudios postobligatorios que cursa la persona encuestada en el momento de la realización del cuestionario. Esta variable agrupa a los y las jóvenes según si hacen Ciclos Formativos de Grado Medio o Bachillerato ${ }^{3}$.

La variable «trayectoria educativa previa» se construyó a partir de 14 preguntas del cuestionario relativas a la trayectoria del joven durante la educación secundaria obligatoria. Éstas hacían referencia tanto a aspectos objetivos de dichas trayectorias, como las notas o las repeticiones de curso, como a aspectos subjetivos o vivenciales. Con el objetivo de reducir estas variables a clústeres que agrupasen tipos similares de trayectorias entre los jóvenes se calculó un análisis factorial que, mediante el método de extracción de componentes principales varimax ${ }^{4}$ redujo las 14 preguntas del cuestionario a 4 componentes. Estos componentes guardan correspondencia teórica con las dimensiones que la literatura previa ha identificado como centrales en la experiencia escolar (Appleton et al., 2008): el sentido de utilidad que él o la joven atribuye al instituto, los resultados y facilidad percibida con los requerimientos académicos, la dimensión conductual y de aceptación o resistencia a la norma escolar, y la sensación de bienestar o malestar en la escuela.

Tal y como se observa en la tabla I, este modelo de 4 componentes explica el 60,12\% de la varianza de las variables que lo forman. Asimismo, el estadístico de adecuación muestral KMO es de 0,807 y la significación de la prueba de esfericidad de Bartlett es menor a 0,05, lo que indica que el modelo es estadísticamente pertinente.

3 Cabe subrayar que, debido al planteamiento de la investigación, ésta se centra en jóvenes que ya están cursando estudios postobligatorios. Por ello, este análisis excluye a aquellos y aquellas que, después de los estudios obligatorios, no siguieron ninguna de estas dos opciones formativas, sea porque optaron por otras vías como programas de formación inicial, sea porque abandonaron los estudios.

4 Este método de rotación optimiza que las distintas preguntas incluidas en el modelo saturen en un solo componente. 


\section{Tabla I. Componentes del modelo e ítems que los forman, \% varianza explicada por los componentes y valor de extracción de cada ítem}

\begin{tabular}{|c|c|c|c|}
\hline COMPONENTES & $\%$ DE VARIANZA EXPLICADA & ÍTEM & EXTRACCIÓN \\
\hline \multirow{5}{*}{ Sentido de utilidad } & \multirow{5}{*}{$21,23 \%$} & «En el instituto me aburía» & 0,464 \\
\hline & & «En el instituto sentía que perdía el tiempo» & 0,609 \\
\hline & & «En general las asignaturas me parecían inútiles» & 0,577 \\
\hline & & «Sentía que valía la pena» & 0,574 \\
\hline & & «En general las asignaturas me parecían interesantes» & 0,679 \\
\hline \multirow{4}{*}{ Resultados académicos } & \multirow{4}{*}{$15,70 \%$} & «Notas medias ESO» & 0,643 \\
\hline & & «En general, no me iban muy bien los exámenes» & 0,601 \\
\hline & & «Tenía facilidad para entender el contenido de la mayoría de las asignaturas» & 0,603 \\
\hline & & «Repetición de curso» & 0,417 \\
\hline \multirow{3}{*}{ Comportamiento } & \multirow{3}{*}{$13,21 \%$} & «Recibía a menudo amonestaciones por parte de mis profesores/as» & 0,686 \\
\hline & & «Ponía mucho esfuerzo en mis tareas escolares» & 0,488 \\
\hline & & «Siempre 0 casi siempre me portaba bien en clase» & 0,72 \\
\hline \multirow{2}{*}{ Bienestar } & \multirow{2}{*}{$9,98 \%$} & «En el instituto me sentía solo/a» & 0,733 \\
\hline & & «En el instituto me lo pasaba bien» & 0,622 \\
\hline Total & $60,12 \%$ & & \\
\hline
\end{tabular}

Fuente: elaboración propia.

A partir de las puntuaciones de los y las estudiantes en estas dimensiones ha sido posible realizar una agrupación por clústeres que establece una tipología de trayectorias educativas. Esta tipología, que se despliega en el primer apartado de los resultados, constituye la variable «trayectoria educativa previa» y ubica a cada uno de los y las jóvenes en uno de estos grupos según el tipo de trayectoria en su paso por la educación obligatoria: los/as «buenos/as», los/as «esforzados/as», los/as «liantes/as», y los/ as «desplazados/as».

Por otro lado, las variables de perfil social ubican al alumnado en posiciones específicas teniendo en cuenta los ejes de clase social, género y a generación migratoria:

- Clase social: se obtiene a partir de los valores más altos a las respuestas sobre categoría profesional y el nivel de estudios de los progenitores del alumno o alumna. La categoría profesional se obtiene a partir de la clasificación de los trabajos de los progenitores mediante el International Standard Classification of Occupation (ISCO-08) y el nivel de estudios a partir del Internacional Standard Classification of Education (ISCED). Se han construido cuatro grupos para la variable clase social: clases acomodadas $(6,6 \%)$, clases medias $(49,8 \%)$, clases trabajadoras $(34,5 \%)$ y riesgo de exclusión social $(9,1 \%)^{5}$.

- Género: si bien el cuestionario admitía otras respuestas a la pregunta «Indica tu género», las respuestas válidas a esta pregunta han permitido construir una variable dicotómica: chicas $(57,2 \%)$ y $\operatorname{chicos}(42,8 \%)$.

5 Las clases acomodadas corresponden mayoritariamente con el grupo 1 (directivos) de ISCO-08, las clases medias con los grupos 2 y 3 (profesionales y técnicos y profesionales asociados), las clases trabajadoras con los grupos 5, 7,8 y 9 (trabajadores de servicios y ventas, artesanía y trabajos afines, operadores y ensambladores de plantas y máquinas y ocupaciones elementales) y el grupo de riesgo de exclusión social constituyen situaciones de inactividad, paro, trabajos informales, o enfermedad de los progenitores. Las familias en los grupos 4, 6 y 0 de ISCO-08 se han desestimado por su baja presencia en la muestra. Finalmente, la correspondencia entre las cuatro clases sociales y la clasificación ISCO-08 puede variar en algunos individuos ya que se tuvo en cuenta el nivel de estudios de los progenitores caso por caso para su clasificación 
- Generación migratoria: se conceptualiza a partir de las trayectorias migratorias protagonizadas por los y las jóvenes y sus progenitores. La categoría de autóctonos/ as $(73,8 \%)$ se refiere a los y las jóvenes nacidas y escolarizadas en España que descienden de progenitores nacidos también en España; las segundas generaciones (17,7\%) las forman los y las jóvenes descendientes de personas nacidas en otro país pero que han sido escolarizados en España desde el inicio de la educación obligatoria; las primeras generaciones (8,5\%) incluyen a aquellos y aquellas jóvenes nacidas fuera de España y no escolarizadas en el país antes del inicio de la enseñanza obligatoria.

Finalmente, la estrategia analítica se ha desarrollado de la siguiente manera: en primer lugar, analizando como las variables que forman los factores y clústeres se distribuyen en cada uno de los tipos de trayectorias obtenidos. En segundo lugar, se cruza la tipología de trayectorias con la variable de itinerario para observar cómo los distintos tipos se distribuyen desigualmente entre el Bachillerato y la Formación Profesional. Finalmente, se introducen en el análisis las variables de perfil social para explicar las características de clase, género y generación migratoria de los tipos de trayectoria identificados y para identificar como la clase social media en la relación entre la trayectoria educativa y los itinerarios.

\section{Resultados}

\subsection{Explorando una tipología de trayectorias escolares}

El primer resultado obtenido de la realización del análisis de conglomerados es una tipología de trayectorias educativas conformada por cuatro grupos que hemos denominado de la siguiente manera: los «buenos/as alumnos/as», los «esforzados/as», los «liantes/as» y los «desplazados/as». A continuación, se presentan las principales características de cada uno de estos grupos, que se encuentran resumidas también en la tabla II. Como se ha señalado anteriormente, la tipología propuesta no se basa únicamente en las experiencias de los y las jóvenes en la escuela, sino que incorpora también una dimensión objetiva de los acontecimientos escolares.

El primer grupo, los «buenos/as alumnos/as», reúne a aquel alumnado que presenta una trayectoria considerada 'socialmente' tanto objetiva como subjetivamente exitosa. Este clúster agrupa 332 individuos $(31,7 \%$ de la muestra) y su principal elemento característico es la mayor puntuación en el componente de resultados académicos. En este sentido, son los alumnos con mejores notas durante la ESO, que además sienten que tienen mayor facilidad para desarrollar las tareas escolares, que los exámenes les van bien y, consecuentemente, prácticamente ninguno de ellos ha repetido curso académico. Además, manifiestan un alto sentido de utilidad respecto a «lo escolan» y unas puntuaciones de comportamiento ligeramente por encima de la media. Respecto al componente de bienestar, las respuestas se sitúan de nuevo por encima de la media, es decir, se divierten en la escuela y no se sienten solos o desatendidos en el marco de esta.

El segundo grupo de trayectorias identificadas es el conformado por aquel alumnado denominado como los «esforzados/as». Esta categoría aglutina a aquellos y aquellas jóvenes que, pese a cumplir con los presupuestos escolares, tienen serias dificultades para superar con éxito los diferentes requisitos educativos de la etapa obligatoria. El clúster agrupa 217 individuos (20,7\% de la muestra) y su principal elemento característico es la presencia de unos resultados académicos muy bajos pero un alto cumplimiento de la norma escolar y una valoración positiva de la utilidad de la institución y sus prácticas. Así pues, estos y estas jóvenes pueden definirse como aquellos que, pese a esforzarse y «cumplir» con 
lo demandado, y de presentar niveles de bienestar medios, no consiguen unos resultados consecuentemente positivos.

El tercer grupo está compuesto por jóvenes etiquetados como los/as «liantes/as» y aglutina a aquellos que presentan trayectorias educativas con síntomas claros de fracaso escolar. Este clúster agrupa 202 individuos (19,3\% de la muestra) y su principal elemento característico es la obtención de una muy baja puntuación en el componente de comportamiento. Este dato manifiesta que el grupo, en comparación al resto, se porta peor en la escuela, se esfuerza menos, no tiene sus tareas escolares a tiempo y recibe muchas más amonestaciones. Sus resultados escolares, es decir las notas, están significativamente por debajo de la media. De hecho, es el grupo que suma mayor número de suspensos, que presenta peores notas y que obtiene el porcentaje de repetición más alto. Por último, también presenta un sentido de utilidad de la escuela y sus aprendizajes claramente por debajo de la media.

El cuarto y último grupo de trayectorias es aquel denominado como los/as «desplazados/as». Este clúster agrupa 164 individuos (15,7\% de la muestra), siendo el menos numeroso de los cuatro, y es el único grupo con niveles de bienestar marcadamente por debajo de la media. Incluso el grupo de los/ as «liantes/as» presenta mayores indicadores de bienestar en la escuela. En relación con sus principales elementos característicos, pese a tener unos resultados académicos y una puntuación en comportamiento ligeramente por encima de la media, estos no están a gusto en el instituto ni encuentran útil lo que allí se produce. En otras palabras, cumplen con los estándares académicos, pero no se sienten vinculados con la institución. Por ejemplo, los indicadores de soledad son muy marcados en este grupo.

En definitiva, el análisis realizado permite identificar, tanto de forma teórica como de forma estadística, cuatro grandes grupos de trayectorias escolares que, incorporando, además de la dimensión subjetiva, una dimensión objetiva, son coherentes con los resultados identificados históricamente en la literatura especializada (por ejemplo, en Bonal et al., 2003). En la tabla II, antes de pasar al análisis en profundidad, se puede observar un resumen de las principales puntuaciones obtenidas en cada componente por cada grupo.

\section{Tabla II. Resumen de las características de los grupos de trayectorias}

\begin{tabular}{lccc} 
& COMPONENTE 1: & COMPONENTE 2: & COMPONENTE 3: \\
& "SENTIDO DE UTILIDAD» & "RESULTADOS ACADÉMICOS» & "COMPORTAMIENTO» \\
\hline Buenos /as & Puntuación media (positiva) & Puntuación muy alta & Puntuación media (positiva) \\
\hline Esforzados/as & Puntuación alta & Puntuación muy baja & Puntuación muy alta \\
\hline Liantes/as & Puntuación media (negativa) & Puntuación muy baja & Puntuación muy baja \\
\hline Desplazados/as & Puntuación baja & Puntuación alta & Puntuación media (positiva) \\
\hline
\end{tabular}

Fuente: elaboración propia.

Nota: Se ha considerado una «puntuación media» cuando los valores obtenidos para el componente se sitúan entre -0.2 y 0.2 , una «puntuación alta» cuando los valores se sitúan entre 0.2 y 0.4 , una «puntuación baja» cuando los valores se sitúan entre - 0.2 y - 0.4 y una «puntuación muy alta»o «muy baja» cuando estos valores superan el 0.4 o el -0.4 respectivamente.

\subsection{La trayectoria educativa: un elemento clave en la transición a la educación postobligatoria}

El objetivo principal propuesto en este artículo es el de identificar el rol que juega la trayectoria educativa previa a la hora de seleccionar un itinerario de educación postobligatoria u otro. En este sentido, 
el presente apartado trata de poner en relación la tipología de trayectorias presentada en el apartado anterior con la elección de una opción formativa concreta. En la tabla III se pueden observar los principales resultados obtenidos.

\section{Tabla III. Itinerario de educación postobligatoria escogido según grupos de trayectorias}

\begin{tabular}{lccccc} 
& $\begin{array}{c}\text { CLÚSTER 1: } \\
\text { BUENOS/AS }\end{array}$ & $\begin{array}{c}\text { CLÚSTER 2: } \\
\text { ESFORZADOS/AS }\end{array}$ & $\begin{array}{c}\text { CLÚSTER 3: } \\
\text { LIANTES/AS }\end{array}$ & $\begin{array}{c}\text { CLÚSTER 4: } \\
\text { DESPLAZADOS/AS }\end{array}$ & TOTAL \\
\hline Bachillerato* & $84,6 \%$ & $37,8 \%$ & $36,1 \%$ & $69,5 \%$ & $60,1 \%$ \\
\hline CFGM $^{*}$ & $15,4 \%$ & $62,2 \%$ & $63,9 \%$ & $30,5 \%$ & $39,9 \%$ \\
\hline Total & $100,0 \%$ & $100,0 \%$ & $100,0 \%$ & $100,0 \%$ & $100,0 \%$ \\
\hline
\end{tabular}

Fuente: elaboración propia.

$* \mathrm{p} \leq 0,01$. Valor de V de Cramer para la variable «Itinerario» $=0,45$.

El primer elemento que se extrae del análisis es la predominancia del Bachillerato como opción formativa muy mayoritaria dentro de la categoría de los «buenos alumnos/as». Casi un 85\% del alumnado con trayectorias académicas exitosas optan por esta opción formativa, siendo los que escogen los CFGM una clara minoría (15\%). Esto pone de relieve un elemento altamente señalado por la literatura (Jacovkis, Montes y Manzano, 2020; Tarabini, Castejón y Curran, 2020): la existencia de una concepción intensamente desigual entre ambos itinerarios, siendo el Bachillerato la opción considerada como «idónea» para las y los buenos estudiantes.

Por otro lado, tanto los «esforzados/as» como los «liantes/as» presentan tendencias totalmente opuestas a los «buenos alumnos/as». Ambos grupos presentan una mayor presencia en los CFGM (62\% y $64 \%$ respectivamente), si bien los datos parecen indicar que la lógica de elección que hay detrás de cada grupo diverge substancialmente. En este sentido, los «esforzados/as» optarían por el itinerario vocacional debido a la interiorización de una sensación de incapacidad a la hora de desarrollar exitosamente las competencias requeridas por el Bachillerato. Así bien, a pesar de que cumplen con los requisitos normativos, su bajo rendimiento académico se erige como una losa infranqueable. Una explicación de estos datos sería que aquí operen elementos institucionales y relacionales tales como las expectativas del profesorado (Tarabini, Castejón y Curran, 2020) o los sesgos en los mecanismos de orientación, ayudando a este proceso de reducción del autoconcepto y de las expectativas del alumnado (Jacovkis, Montes y Manzano, 2020). Por su parte, los «liantes/as» no solo presentan resultados académicos también negativos, sino que además muestran una clara oposición a la cultura escolar, hecho que explicaría su no continuidad dentro del itinerario académico. En esta línea, no solo se verían incapaces de realizar el Bachillerato, sino que, en la mayoría de los casos, esta sería una opción que ni tan solo se plantean como deseada.

Por último, el grupo de los «desplazados/as» presenta resultados muy interesantes ya que, a pesar de mostrar un sentimiento de bienestar muy bajo optan, de forma casi mayoritaria, por continuar con el itinerario académico (69,5\%). Es decir, a pesar de no estar cómodos en la escuela, deciden seguir en ella. Esto se podría explicar por los mismos motivos que llevarían a los «esforzados/as» a escoger el itinerario profesionalizador. En primer lugar, una arquitectura y una cultura escolar que, mediante las interacciones cotidianas con su profesorado - por ejemplo-, orienta al alumnado con «buenos» resultados al Bachillerato, independientemente de los elementos subjetivos relativos al bienestar que acompa- 
ñen a la trayectoria En segundo lugar, una alta estratificación educativa que considera los CFGM como una opción formativa de segunda categoría que no brinda oportunidades de calidad.

\subsection{Trayectorias escolares, itinerarios postobligatorios y variables estructurales}

Una vez identificada la relación entre la trayectoria escolar y la elección del itinerario de educación postobligatoria, es interesante observar cómo las variables estructurales median tanto la composición de estos grupos como la misma elección de itinerario. La tabla IV sintetiza los principales resultados observados.

\section{Tabla IV. Distribución de las variables estructurales (género, generación migratoria y clase social) según grupos de trayectorias}

\begin{tabular}{|c|c|c|c|c|c|c|}
\hline & & & & & \\
\hline & & $\begin{array}{l}\text { CLÚSTER 1: } \\
\text { BUENOS/AS }\end{array}$ & $\begin{array}{c}\text { CLÚSTER 2: } \\
\text { ESFORZADOS/AS }\end{array}$ & $\begin{array}{l}\text { CLÚSTER 3: } \\
\text { LIANTES/AS }\end{array}$ & $\begin{array}{c}\text { CLÚSTER 4: } \\
\text { DESPLAZADOS/AS }\end{array}$ & TOTAL \\
\hline \multirow{2}{*}{ Género* } & Chico & $39,1 \%$ & $39,6 \%$ & $59,7 \%$ & $32,9 \%$ & $42,8 \%$ \\
\hline & Chica & $60,9 \%$ & $60,4 \%$ & $40,3 \%$ & $67,1 \%$ & $57,2 \%$ \\
\hline \multirow{3}{*}{$\begin{array}{l}\text { Generación } \\
\text { migratoria* }\end{array}$} & Autóctono & $87,3 \%$ & $55,8 \%$ & $71,8 \%$ & $72,6 \%$ & $73,8 \%$ \\
\hline & $2^{\text {as }}$ Generaciones & $10,2 \%$ & $27,6 \%$ & $16,3 \%$ & $21,3 \%$ & $17,7 \%$ \\
\hline & $1^{\text {as }}$ Generaciones & $2,4 \%$ & $16,6 \%$ & $11,9 \%$ & $6,1 \%$ & $8,5 \%$ \\
\hline \multirow{4}{*}{$\begin{array}{l}\text { Clase } \\
\text { Social }^{*}\end{array}$} & Clases acomodadas & $10,7 \%$ & $4,4 \%$ & $2,1 \%$ & $6,5 \%$ & $6,6 \%$ \\
\hline & Clases Medias & $58,4 \%$ & $34,8 \%$ & $46,1 \%$ & $56,1 \%$ & $49,8 \%$ \\
\hline & Clases trabajadoras & $26,6 \%$ & $46,6 \%$ & $35,8 \%$ & $33,5 \%$ & $34,5 \%$ \\
\hline & Riesgo de exclusión social & $4,3 \%$ & $14,2 \%$ & $16,1 \%$ & $3,9 \%$ & $9,1 \%$ \\
\hline
\end{tabular}

Fuente: elaboración propia.

$* \mathrm{p} \leq 0,01$. V de Cramer para la variable «género» $=0,19 . \mathrm{V}$ de Cramer para la variable «generación migratoria» $=0,20$. V de Cramer para la variable «clase social» $=0,17$.

En este sentido, lo primero que se identifica es como el grupo de trayectorias de los buenos alumnos/as está conformado por una mayoría de chicas (61\%), autóctonas $(87 \%)$ y provenientes de entornos de clase media (58\%). Además, tienen el mayor porcentaje de alumnado de clases acomodadas de toda la muestra (10,7\%). A su vez, este grupo comparte características altamente similares con el grupo de los «desplazados/as», el otro grupo que presenta buenos indicadores en el componente de «resultados académicos». No obstante, el principal elemento que distingue ambos grupos es que los «desplazados/as» es un grupo ligeramente más feminizado (67\%), con una mayor presencia de las clases trabajadoras $(33,5 \%)$ y una menor presencia de alumnado autóctono, siendo el incremento observado en el alumnado de segunda generación (21,3\%). Los perfiles sociales que predominan en estos tipos de trayectoria son los que, como ha demostrado ampliamente la literatura sociológica, acostumbran a gozar de unas relaciones más afines con la escuela -expectativas positivas por parte del profesorado, actitudes que se ven recompensadas por la institución, etc.-, relaciones que influyen en las elecciones postobligatorias desarrolladas por ambos grupos.

Por su parte, los «esforzados/as» y los «liantes/as» presentan perfiles más diferenciados en relación con los otros dos grupos identificados. En este sentido, los «esforzados/as» presentan una mayor proporción de jóvenes de origen migrante, tanto de primeras generaciones $(17 \%)$ como de segundas generaciones $(28 \%)$. Además, es el grupo que presenta más alumnado de clase trabajadora (47\%). Esto reafirma la idea predominante detrás de este grupo, el «esfuerzo sin recompensa», puesto que recoge a los grupos socialmente más distantes de la cultura escolar hegemónica. 
En relación con los «liantes/as», este se erige como el único grupo masculinizado de la muestra (con un $60 \%$ del total). Siguiendo con su caracterización, predominan los autóctonos por encima de cualquier otro grupo $(72 \%)$ y, en términos de perfil social, se evidencia una alta heterogeneidad. La clase social más dominante es la clase media (46\%) pero también presenta el porcentaje más elevado de alumnado de entornos sociales en riesgo de exclusión (16\%). Esto pone de relieve la naturaleza diversa existente detrás de este tipo de trayectorias.

En definitiva, el análisis desarrollado muestra como existe una clara correlación entre los grupos de trayectorias identificadas y las probabilidades de ejercer una transición hacia un itinerario formativo u otro. En ese sentido, los datos obtenidos son claros: los «buenos/as alumnos/as» tienen más del doble de opciones de matricularse en la vía académica, el Bachillerato, que sus compañeros «esforzados/as» o «liantes/as». Dicho de otra manera, estos grupos tienen hasta casi cuatro veces más probabilidades de acabar realizando una formación profesional que los primeros. Este hecho pondría de relieve la importancia ejercida por la trayectoria escolar y aportaría una nueva evidencia a la desmitificación de la retórica existente sobre la «libre elección» en la transición a los estudios postobligatorios (Furlong, 2009).

\subsection{Mismas trayectorias, diferentes destinos: el efecto mediador de la clase social}

Por último, se ha analizado cómo se distribuyen los distintos grupos de trayectorias en los dos itinerarios para cada una de las clases sociales identificadas. La fragmentación de la muestra que implica este ejercicio, invalida la significatividad estadística para las clases acomodadas y para el grupo en riesgo de exclusión social pero no invalida las conclusiones para las clases medias y trabajadoras. Por este motivo, en este análisis se ha trabajado únicamente con la submuestra de clases medias y trabajadoras. La lectura principal se sintetiza en la tabla siguiente (tabla V) en la que se observa cómo tanto las trayectorias como la clase social de los jóvenes influyen en el itinerario educativo que éstos terminan adoptando.

\section{Tabla V. Distribución de los grupos de trayectorias según clase social simplificada y elección del itinerario de educación postobligatoria}

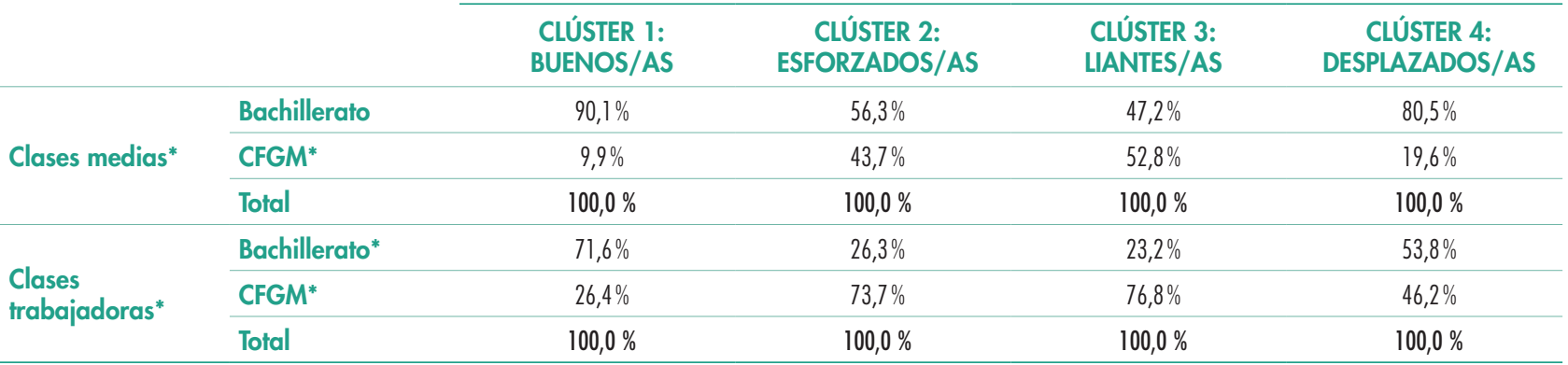

Fuente: elaboración propia.

$* \mathrm{p} \leq 0,01 . \mathrm{V}$ de Cramer para la variable «clases medias» $=0,406 . \mathrm{V}$ de Cramer para la variable «clases trabajadoras» $=0,434$.

El análisis desarrollado pone de relieve que la clase social sigue siendo un elemento mediador relevante. Independientemente del grupo al que se adscriba la trayectoria, aquellos que provienen de entornos sociales más favorecidos tienen una mayor probabilidad de acceder al Bachillerato. Y viceversa, los que provienen de entornos de clase trabajadora acaban, en mayor proporción, accediendo a estudios profesionalizadores. 
En conclusión, estos datos muestran que, si se introduce en el análisis un efecto mediador, existe una triple relación entre la trayectoria escolar, el itinerario escogido en la transición a la educación postobligatoria y las variables estructurales, específicamente el perfil social del alumnado. En este sentido, se observa como ambos elementos -perfil social y trayectoria escolar- operan simultáneamente acrecentando la desigualdad en el momento de la transición. El alumnado proveniente de entornos acomodados continua en mayor proporción en el itinerario académico -obteniendo hasta 30 puntos porcentuales más- que el alumnado que, a pesar de haber desarrollado la misma trayectoria, proviene de entornos de clase trabajadora menos favorecidos. Esto es especialmente relevante en el grupo de los «esforzados/as», fenómeno que pone de relieve, en primer lugar, el papel de las propias expectativas (Jacovkis, Montes y Manzano, 2020) y, en segundo lugar, el rol que ejercen los capitales como red de seguridad ante las situaciones de adversidad (Ball, 2003).

\section{Conclusiones}

El análisis de las transiciones educativas lleva décadas ganando terreno en el campo de la investigación sociológica, convirtiéndose en los últimos años en un objeto de estudio de relevancia central. Las elecciones educativas y las transiciones resultantes son procesos socialmente integrados, donde la agencia de los y las jóvenes se encuentra informada y limitada por las dimensiones sociales de la desigualdad (Archer, et al., 2007; Tarabini e Ingram, 2018). En otras palabras, tal y como se ha señalado al inicio del artículo, existe una estructura de oportunidades que refleja las posiciones estructuralmente desiguales en las biografías, las experiencias y las elecciones de los y las jóvenes, repercutiendo en las posibilidades, pero también en las barreras, que estos y estas encuentran a lo largo de sus trayectorias educativas.

Así pues, comprender el papel de la escuela y del sistema educativo como parte fundamental de la estructura de oportunidades es prioritario si se desea abordar la desigual configuración de las trayectorias formativas. La investigación previa (Cuconato, et al., 2015; Dubet, 2010) ha demostrado que la experiencia desarrollada en el seno de la institución educativa tiene un impacto diferencial, que está mediado por el origen social del alumnado. Entender bajo qué supuestos los y las jóvenes configuran sus trayectorias escolares es básico para abordar cuestiones como la distribución de los y las jóvenes en los diferentes itinerarios -académico o profesional- de la educación postobligatoria, entre otros aspectos.

Partiendo de este marco, el objetivo del presente artículo ha sido el de comprender como se constituyen las trayectorias escolares de los y las jóvenes, poniendo de relieve el rol clave jugado por la institución escolar a la hora de construir oportunidades desiguales de transición a la educación postobligatoria. Con este propósito, se ha desarrollado un análisis factorial y un análisis de conglomerados cuyo resultado ha sido la obtención de una tipología de trayectorias escolares conformada por cuatro grupos: los «buenos/ as», los «esforzados/as», los «liantes/as» y los «desplazados/as». Estos grupos, constituidos en base a cuatro grandes dimensiones de la experiencia escolar -utilidad de la escuela, resultados educativos, comportamiento y bienestar escolar- muestran como los y las jóvenes encuestados han vivido su paso por la institución escolar de forma muy diferente, repercutiendo esta experiencia en su futura transición. Por otro lado, la composición social de dichos grupos -en términos de perfil social, género y generación migratoria- pone de relieve el efecto mediador que continúan ejerciendo las variables estructurales a la hora de desarrollar un tipo de trayectoria u otra. 
Los «buenos/as alumnos/as» se caracterizan por una alta vinculación escolar y un buen rendimiento académico. Son alumnos modélicos, aquellos que siguen las directrices del orden escolar, y que, en consecuencia, presentan trayectorias altamente exitosas en términos académicos. Del mismo modo, no sorprende que sea el grupo con mayor presencia de clases acomodadas y de alumnado autóctono, fenómeno que explica parte de este «éxito».

Por su parte, el resto de los grupos presentan diferentes posicionamientos dentro de la escala de grises de la experiencia escolar. Los «liantes/as» se caracterizan por su poca vinculación conductual mientras que los «esforzados/as» por su baja vinculación cognitiva (Fredricks, et al., 2004). Ambos grupos acaban «fracasando», si bien los motivos de dicho fracaso son de naturaleza bien distinta. Además, su perfil social es totalmente opuesto. Mientras que los «liantes/as» presentan una situación heterogénea pero socialmente acomodada, donde el elemento que más destaca es la acentuada masculinización del grupo, los «esforzados/as» todo lo contrario. Son el grupo que aglutina mayor distancia social -clase trabajadora y en riesgo de exclusión social- y cultural -alumnado no autóctono- con la escuela. Finalmente, el grupo de los «desplazados/as», a pesar de obtener indicadores educativos y sociales similares a los de los buenos/ as alumnos/as, se caracteriza por la dificultad de encontrar su lugar en la institución escolar. En este sentido, resulta interesante observar como a pesar de ser un grupo que lo pasa mal en la escuela, presentan mayoritariamente trayectorias de incorporación a la vía más prestigiosa, el bachillerato. Esto pondría de relieve la importancia ejercida por los mecanismos de path-dependence ${ }^{6}$.

Además de la construcción de esta tipología, el análisis desarrollado aporta otros dos resultados relevantes. En primer lugar, que el itinerario «escogido» en la transición a la educación postobligatoria está claramente correlacionado con las trayectorias escolares previas, y que estas pueden limitar o ampliar las probabilidades de acabar en un itinerario profesional o académico. En segundo lugar, que el origen social es una variable que tiene un claro efecto mediador entre la trayectoria escolar previa y el itinerario de educación postobligatoria: entre jóvenes que han tenido un mismo tipo de trayectoria, aquellos y aquellas que provienen de entornos más acomodados tienen mayores probabilidades de transitar hacia el Bachillerato, mientras que los y las jóvenes de entornos de clase trabajadora, aun estando en trayectorias consideradas «exitosas», tienden a transitar, en mayor medida, hacia el itinerario profesional.

En definitiva, las conclusiones de este artículo confirman los hallazgos previos: las trayectorias educativas -que incorporan tanto los acontecimientos objetivos como una dimensión subjetiva referente a la experiencia escolar- juegan un papel relevante en la transición a la educación postobligatoria. El análisis presentado ha permitido constatar cuantitativamente que las trayectorias previas y la transición a la postobligatoria son construcciones en las que intervienen tanto factores estructurales, como institucionales y subjetivos. En otros análisis vinculados al proyecto se analizan las interacciones con los actores significativos para estos jóvenes (familias, compañeros/as, profesorado), permitiendo identificar de qué manera estos son un elemento clave en la construcción de las trayectorias educativas durante la educación obligatoria y cómo estas interacciones contribuyen a explicar las transiciones a la educación postobligatoria. Solo desde esta óptica relacional, que incorpore también el efecto mediador ejercido por las variables estructurales, se podrán comprender las desigualdades subyacentes a las transiciones educativas de una forma integral que permita abordarlas en consecuencia.

6 La patb-dependence, o dependencia del camino, hace referencia a un fenómeno altamente explorado en las Ciencias Sociales y Económicas que expone que determinados resultados son producto de las elecciones, decisiones y situaciones desarrolladas en el pasado y no solo de las condiciones del momento. Dicho de otra manera, y aplicado al campo que aquí nos atañe -las transiciones educativas- vendría a identificar y explicar los procesos de «no elección», de inercia institucional, bajo los cuales algunos y algunas jóvenes enmarcan su paso entre etapas educativas. 


\section{Referencias bibliográficas}

Appleton, James J.; Christenson, Sandra L. y Furlong, Michael J. (2008): "Student Engagement with School: Critical Conceputal and Methodological Issues of the Construct". Psychology in the Schools, 45 (5), 369-386. DOI: https://doi.org/10.1002/pits.20303.

Archer, Louise; Hollingworth, Sumi y Halsall, Anna (2007): “University's not for Me- I'm a Nike Person': Urban, Working-Class Young People's Negotiations of 'Style', Identity and Educational Engagement”. Sociology, 41 (2), 219-237. DOI: https://doi.org/10.1177/0038038507074798.

Ball, Stephen (2003). Class Strategies and the Education Market: The middle Classes and Social Advantage. London: Routledge.

Bernardi, Fabrizio y Requena, Miguel (2010). "Desigualdad y puntos de inflexión educativos: el caso de la educación post-obligatoria en España”. Revista de Educación, Número Ext, 93-118.

Bernardi, Fabrizio y Cebolla-Boado, Hector (2014): "Previous School Results and Social Background: Compensation and Imperfect Information in Educational Transitions". European Sociological Review, 30 (2), 207-217. DOI: https://doi.org/10.1093/esr/jct029.

Bernstein, Basil (1988). Clases, códigos y control. Vol 2. Madrid: Akal.

Bonal, Xavier; Alegre, Miquel À.; González, Isaac; Herrera, Diego; Rovira, Marta y Saurí, Enric (2003). Apropiacions escolars: usos i sentits de l'educació obligatòria en l'adolescència. Barcelona: Editorial Octaedro.

Bonizzoni, Paola; Romito, Marco y Cavallo, Cristina (2014): “Teachers' Guidance, Family Participation and Track Choice: The Educational Disadvantage of Immigrant Students in Italy". British Journal of Sociology of Education, 37 (5), 702-720. DOI: https://doi.org/10.1080/01425692.2014.982860.

Calero, Jorge (2008). Sociedad desigual, ¿educación desigual? Sobre las desigualdades en el sistema educativo español. Madrid: CIDE, Ministerio de Educación, Política Social y Deporte.

Castejón, Alba (2017). Expectativas docentes, agrupamiento del alumnado y segregación escolar. Una etnografía en entornos de alta complejidad social en Cataluña. Tesis Doctoral. Departament de Pedagogia Sistemàtica i Social. Universitat Autònoma de Barcelona.

Cuconato, Morena; du Bois-Reymond, Manuela y Lunabba, Harry (2015): "Between Gate-Keeping and Support: Teachers' Perception of their Role in Transition". International Journal of Qualitative Studies in Education, 28 (3), 311-328. DOI: https://doi.org/10.1080/09518398.2014.987854.

Cuconato, Morena y Walther, Andreas (2015): “"Doing Transitions' in Education”. International Journal of Qualitative Studies in Education, 28 (3), 283-296. DOI: https://doi.org/10.1080/09518398.2014.987851

Curran, Marta (2017). ¿Qué lleva a los jóvenes a dejar los estudios? Explorando los procesos de (des)vinculación escolar desde una perspectiva de clase y género. Tesis doctoral. Departament de Sociologia. Universitat Autònoma de Barcelona.

Dubet, François (2010). Sociología de la experiencia. Madrid: Centro de Investigaciones Sociológicas.

Dubet, François, Martucelli, Danilo (1998). En la escuela. Sociología de la experiencia escolar. Buenos Aires: Editorial Losada. 
Elias, Marina y Daza, Lidia (2017): “CCómo deciden les jóvenes la transición a la educación postobligatoria? Diferencias entre centros públicos y privados-concertados". RASE: Revista de la Asociación de Sociología de la Educación, 10 (1), 5-22. DOI: https://doi.org/10.7203/rase.10.1.9135.

Fredricks, Jennifer; Blumenfeld, Phyllis y Paris, Alison (2004): "School Engagement: Potential of the Concept, State of the Evidence". Review of Educational Research, 74 (1), 59-109.

Furlong, Andy (2009): "Revisiting Transitional Metaphors: Reproducing Social Inequalities Under the Conditions of Late Modernity". Journal of Education and Work, 22 (5), 343-353. DOI: https://doi. org/10.1080/13639080903453979.

Gamoran, Adam (1992): "Synthesis of Research: is Ability Grouping Equitable?". Educational Leadership, $50(2), 11-17$.

Garcia, Maribel y Sánchez-Gelabert, Albert (2020): "La heterogeneidad del abandono educativo en las transiciones posobligatorias. Itinerarios y subjetividad de la experiencia escolar". Papers. Revista de Sociologia, 105 (2), 235. DOI: https://doi.org/10.5565/rev/papers.2775.

Grytnes, Regine (2011): "Making the Right Choice! Inquiries into the Reasoning Behind Young People's Decisions about Education”. Young, 19(3), 333-351. DOI: https://doi.org/10.1177/1103308811019 00305 .

Jacovkis, Judith; Montes, Alejandro y Manzano, Martí (2020): “Imaginando futuros distintos. Los efectos de la desigualdad sobre las transiciones hacia la educación secundaria posobligatoria en la ciudad de Barcelona”. Papers. Revista de Sociologia, 105 (2), 279. DOI: https://doi.org/10.5565/rev/papers.2773.

Oakes, Jeannie (1985). Keeping Track: How Schools Structure Inequality. New Haven: Yale University Press.

Pallas, Aaron M. (2003): "Educational Transitions, Trajectories, and Pathways" en Jeylan T. Mortimer y Michael J. Shanahan (eds.): Handbook of the Life Course. DOI: https://doi.org/10.1007/978-0-30648247-2_8.

Pàmies, Jordi (2013): "El impacto de los agrupamientos escolares. Los espacios de aprendizaje y sociabilidad de los jóvenes de origen marroquí en Barcelona". Revista de Educación, 362 (Septiembre-diciembre 2013), 133-158. DOI: https://doi.org/10.4438/1988-592X-RE-2011-362-156.

Parsons, Samantha y Hallam, Sue (2014): "The Impact of Streaming on Attainment at Age Seven: Evidence from the Millennium Cohort Study". Oxford Review of Education, 40 (5), 567-589. DOI: https:/ / doi.org/10.1080/03054985.2014.959911.

Slavin, Robert E. (1990): "Achievement Effects of Ability Grouping in Secondary Schools: A Best-Evidence Synthesis". Review of Educational Research, 60(3), 471-499. DOI: https://doi. org/10.3102/00346543060003471.

Tarabini, Aina (2019). The Conditions for School Success. Examining Educational Exclusion and Dropping Out. Palgrave Macmillan. DOI: https://doi.org/https://doi.org/10.1007/978-3-030-02523-6. 
Tarabini, Aina; Castejón, Alba y Curran, Marta (2020): “Capacidades, hábitos y carácter: atribuciones docentes sobre el alumnado de Bachillerato y Formación Profesional”. Papers. Revista de Sociologia, 105 (2), 211. DOI: https://doi.org/10.5565/rev/papers.2778.

Tarabini, Aina y Jacovkis, Judith (2019): “Transicions a l'educació secundària postobligatòria a Catalunya” en Jordi Riera (ed.): Reptes de l'educació a Catalunya. Anuari 2018. Barcelona: Fundació Jaume Bofill.

Tarabini, Aina y Ingram, Nicola (2018). Educational Choices, Transitions and Aspirations in Europe: Systemic, Institutional and Subjective Challenges. Londres: Routledge.

Tikkanen, Jenni; Bledowski, Piotr y Felczak, Joanna (2015). "Education Systems as Transition Spaces". International Journal of Qualitative Studies in Education, 28 (3), 297-310. DOI: https:/ / doi.org/10.1080/0 9518398.2014.987853.

Van Houtte, Mieke y Demanet, Jannick (2016). "Teachers' Beliefs about Students, and the Intention of Students to Drop out of Secondary Education in Flanders". Teaching and Teacher Education, 54, 117-127. DOI: https://doi.org/10.1016/j.tate.2015.12.003.

Walther, Andreas; Warth, Annegret; Ule, Mirjana y du Bois-Reymond, Manuela (2015): “Me, My Education and I': Constellations of Decision-Making in Young People's Educational Trajectòries”. International Journal of Qualitative Studies in Education, 28 (3), 349-371. DOI: https://doi.org/10.1080/095183 98.2014.987850.

Wyn, Johanna y Dwyer, Peter (2002): “New Patterns of Youth Transition in Education”. International Social Science Journal, 52 (164), 147-159. DOI: https://doi.org/https://doi.org/10.1111/1468-2451.00247.

\section{Notas biográficas}

Alba Castejón es Doctora en Educación por la Universitat Autònoma de Barcelona (2017) donde actualmente es investigadora postdoctoral (Departamento de Teorías de la Educación y Pedagogía Social). Su labor investigadora se ha enmarcado principalmente en los grupos de investigación GAPEF-UAB y GEPS-UAB, y actualmente es miembro del equipo del proyecto I+D EDUPOST16. Sus principales intereses de investigación son las desigualdades educativas y su relación con las políticas educativas, las prácticas de los centros educativos y del profesorado, y las experiencias escolares de los y las jóvenes.

Alejandro Montes es Doctor en Sociología de la Educación por la Universidad Autónoma de Barcelona y miembro del grupo de investigación en Globalización, Educación y Políticas Sociales (GEPS-UAB). Forma parte del equipo investigador del proyecto I+D 'La construcción de las oportunidades educativas post-16. Un análisis de las transiciones a la educación secundaria post-obligatoria en contextos urbanos' (http://edupost16.es/). Su investigación se centra en el análisis de las trayectorias y transiciones educativas desde el prisma de la desigualdad social, si bien, también ha trabajado en ámbitos tales como el estudio del abandono escolar prematuro, el análisis de la segregación escolar o el estudio de las barreras de acceso a la educación superior. 
Martí Manzano graduado en Sociología (2016) y máster en Políticas Sociales, Trabajo y Bienestar (2017), ambos títulos en la Universidad Autónoma de Barcelona. Actualmente está realizando un doctorado como Investigador Predoctoral en Formación (PIF) del Departamento de Sociología de la UAB. Es miembro del GEPS (Globalización, Educación y Políticas Sociales) desde 2015 y del GIPE (Grupo Interdisciplinario de Políticas Educativas) desde 2018. Ha participado en investigaciones dentro y fuera de la universidad sobre abandono y oportunidades educativas y planificación de política educativa, actualmente forma parte del equipo principal del proyecto Edupost16. Sus áreas de especialización son las desigualdades educativas, vinculadas con la clase social y el país de origen, el análisis de políticas educativas y la innovación escolar. Su tesis doctoral investiga las transiciones educativas post16 de jóvenes descendientes de migrantes en contextos socio-educativos altamente segregados. 